\title{
Dosimetric Characteristics of 6 MV Medical Linac at BAEC
}

\section{Sudeb Kumar Roy', Pretam Kumar Das ${ }^{1}$, Rajada Khatun², Md. Ashikur Rahman ${ }^{3}$, Shirin Akter ${ }^{2}$, Tushar Kumar1, Mohammad Monjur Ahasan³}

\author{
${ }^{1}$ Department of Physics, Pabna University of Science and Technology, Pabna, Bangladesh \\ ${ }^{2}$ Medical Physics Division, Atomic Energy Centre, Dhaka, Bangladesh \\ ${ }^{3}$ Institutes of Nuclear Medical Physics, Atomic Energy Research Establishment, Savar, Dhaka, Bangladesh \\ Email: *shirin_apece@yahoo.com
}

How to cite this paper: Roy, S.K., Das, P.K., Khatun, R., Rahman, Md.A., Akter, S., Kumar, T. and Ahasan, M.M. (2021) Dosimetric Characteristics of 6 MV Medical Linac at BAEC. International Journal of Medical Physics, Clinical Engineering and Radiation Oncology, 10, 38-46.

https://doi.org/10.4236/ijmpcero.2021.101004

Received: October 25, 2020

Accepted: February 6, 2021

Published: February 9, 2021

Copyright $\odot 2021$ by author(s) and Scientific Research Publishing Inc. This work is licensed under the Creative Commons Attribution International License (CC BY 4.0).

http://creativecommons.org/licenses/by/4.0/

\begin{abstract}
Dosimetric characteristic is one of the essential parameters of a medical linear accelerator (LINAC), which must be obtained before clinical use. The dosimetric characteristics for $6 \mathrm{MV}$ photon beam were measured and compared with the corresponding published data. The study was done using a Varian linear accelerator (Model Clinac-iX) at the Institute of Nuclear Medical Physics (INMP), AERE, Savar, Dhaka, under the Bangladesh Atomic Energy Commission (BAEC). The data is taken for 10 field sizes $(2 \times 2,3 \times$ $3,5 \times 5,7 \times 7,10 \times 10,15 \times 15,20 \times 20,25 \times 25,30 \times 30$ and $\left.40 \times 40 \mathrm{~cm}^{2}\right)$ at same conditions. The measured Percent Depth Dose (PDD) curves were obtained for $6 \mathrm{MV}$ photon beams with the field as mentioned above and compared with the calculated PDD curves. The measured depth dose $\left(D_{\max }\right)$ for reference field size (FS) $10 \times 10 \mathrm{~cm}^{2}$ is $15.99 \mathrm{~mm}$, and the PDD at $10 \mathrm{~cm}$ depth $\left(D_{10}\right)$ is $66.87 \%$ for $6 \mathrm{MV}$ photon energies that are found to be compatible with the published report BJR supplement 25. The measured PDD curves for photon energies show a good agreement with the standard PDD curves. The photon beam dosimetry data found in the current study are compatible and all the tolerances are within the clinically acceptable tolerance limit.
\end{abstract}

\section{Keywords}

Linac Machines, 3D Water Phantom, Ionization Chamber, Electrometer

\section{Introduction}

Dosimetric characteristics are the most important parameter of a linear accelerator (LINAC) which must be obtained prior to clinical use. Most of the devel- 
oped countries are used basic treatment for linear accelerator. They can produce photon beams, electron beam or both depending on the model used. A linear accelerator can produce high energy x-rays to ensure a tumor's shape and destroy cancer cells while canning surrounding normal tissue [1]. The Photon beams are produced with a wide spectrum of energies ranging up to the maximum energy of the electrons striking the target, with a peak at about half this maximum value [2] [3] [4]. The linear accelerators have the advantage that the photon beam dosimetric is more penetrating within the cancer cell, a diverse choice of beam energy, a smaller penumbra edge to the beam, and they provide a high dose rate and calculate accurate dose measurement [2].

The LINAC can be used for different radio-therapies (treatment) such as surgery, chemotherapy, and target therapy after completion of some satisfactory scientific methods called as pre-commissioning testing [5]. Therefore, it is essential to have a minimum data set which includes percentage depth dose (PDD), Open Beam Profile (inline, cross line \& diagonal) and Beam Output factors for a series of Field Sizes (FSs) are the important dosimetric data that require during the commissioning period of a medical linear accelerator (LINAC). In this study, one basic dosimetric parameter like PDD for $6 \mathrm{MV}$ photon beams with different FSs was measured. These measurements have been performed in a three dimensional (3D) computer controlled water phantom using two ionization chambers (S/N: 15050 and 15051) for the Varian Clinac iX linear accelerator machine at the Institute of Nuclear Medical Physics, Savar, Dhaka. The data obtained during the initial commissioning of LINAC can be treated as the standard data for clinical purpose. The scientific methods used for commissioning of modern Linear Accelerator are really a time-consuming procedure and need dedication in work [5]. In this work, the PDDs were compared for measured data. The objective of this work is to investigate the Varian technology evolution via a comparative study of percentage depth dose (PDD) and especially the build-up region for accelerators Varian Clinac iX. The main objective of this research, using present knowledge of relative dosimetry of photon based on different international literature, reports and guidelines and approach for possible suitable discuss their response in different fields' size photon. In this present research, different sizes of active volume chambers, water phantom (1D, 3D) were used. A significant amount of data is accumulated for this thesis.

This data set gives the complete output factor to be used in configuring the treatment planning system at SSD $=100 \mathrm{~cm}$ and Depth $=10 \mathrm{~cm}$. On the other hand, for the edge detector and the other two ionization chambers measurement readings were taken for field sizes of square widths from $10 \mathrm{~cm} \times 10 \mathrm{~cm}$ for a total of 10 square field sizes.

For the two types of ionization chamber and two phantoms used and the measurement combination from two possible SSDs and Depths and also with the choice of one types of field collimation, the whole package constitutes a significantly huge data set. These data and their analyses could constitute a valuable 
addition toward understanding photon beam radiation measurements in medical linacs. The main advantage of removing maximum and minimum flatness and symmetry was on increasing dose and the increasing of dose rate is approximately $80 \%$ to $20 \%$ for field size of $10 \times 10 \mathrm{~cm}^{2}$ and it is approximately $100 \%$ for field size of $10 \times 10 \mathrm{~cm}^{2}$.

\section{Materials and Methods}

The measurements were conducted using Varian Clinac iX (Manufacture: Varian Medical System, USA) (Varian Oncology) at the Institute of Nuclear Medical Physics, Bangladesh Atomic Energy Commission. For this study, 1D and 3D water phantom, ionization chambers, and electrometers are used. The ionization chamber is put in a water phantom, and is connected with a myQA accept software on a computer via an optical cable. The photon beam field area is set at 10 $\times 10 \mathrm{~cm}^{2}$. Later, the SSD was set at a distance of $90 \mathrm{~cm}$ and the energy of $6 \mathrm{MV}$. The ionization chamber's depth in a water phantom is positioned with a depth of $5 \mathrm{~cm}$ from the water surface. The beam focus would set on, and PDD and dose profile would be analyzed using the IBA blue phantom-2 software.

The procedure would be repeated by adjusting the SSD, energy, and depth. The variations of SSD are 90,100, and $110 \mathrm{~cm}$. The depth of the water phantom is $5 \mathrm{~cm}, 10 \mathrm{~cm}, 15 \mathrm{~cm}, 20 \mathrm{~cm}$, and $25 \mathrm{~cm}$. The water phantom's depth is created for analysis of percentage depth dose (PDD) and dose profile. Radiation dose profile characteristics of the radiation beam and provide essential information for radiotherapy planning. That curve interprets the distribution of radiation dose relatively on a particular radiation field. Percentage depth dose is the absorbed dose is given on the main depth as a percentage of absorbed doses at a depth of the pointer on the central axis of the area. In order to characterize the dose distribution on the central beam axis in a water phantom, the dose should be normalized to maximum dose [6].

The central axis dose distributions inside the patient or phantom are usually normalized to Dmax $=100 \%$ at a depth of dose maximum Dmax and then referred to as the PDD distributions [7]. Dose distribution at the point in the central axis of the files inside the phantom usually normalized to Dmax as $100 \%$ at a depth of maximum dose, Dmax corresponding to the reference depth. Value percentage depth dose can be defined as a result, in the form of a percentage, the dose absorbed at a certain depth where a depth $d$ is, $d_{0}$ is the reference dose along the beam axis [8].

\section{Data Collection and Analysis}

This data was collected using the clinac iX machine with Eclips 13.7 Version, MyQA Accept software, water phantom, etc. for $6 \mathrm{MV}$ energy, SSD = 100, Dose Rate $=300$, Depth $=10 \mathrm{~cm}$.

Ionization chambers $\mathrm{CC} 13$ is used to output factor analysis and FC65-P is used to analyze the response of chambers in different field sizes concerning 
chamber's active volume. Actually, using Dose-1 electrometer reading in $\mathrm{nC}$, dose for $2 \times 2,3 \times 3,5 \times 5,7 \times 7,10 \times 10,15 \times 15,20 \times 20,25 \times 25,30 \times 30$ and $40 \times 40 \mathrm{~cm}^{2}$ field sizes is measured. The output factor shown in Table 1 is measured using different size volume Chambers and is normalized with $10 \times 10 \mathrm{~cm}^{2}$ field size dose. This data set gives the complete output factor to be used in configuring the treatment planning system at SSD $=100 \mathrm{~cm}$ and Depth $=10 \mathrm{~cm}$. The two ionization chambers measurement readings were taken for field sizes of square widths from $10 \mathrm{~cm} \times 10 \mathrm{~cm}$ for a total of 10 square field sizes.

\section{Results and Discussion}

In the present study, this is a raw data calculation of 10 different field sizes with a $6 \mathrm{MV}$ photons beam that was performed at the Institute of Nuclear Medical Physics (INMP), Bangladesh Atomic Energy Commission. PDDs were measured for various field sizes ranging from $5 \times 5 \mathrm{~cm}^{2}, 10 \times 10 \mathrm{~cm}^{2}, 15 \times 15 \mathrm{~cm}^{2}$ and $20 \times$ $20 \mathrm{~cm}^{2}$. Various parameters such as $D_{\max }(1.6 \mathrm{~cm}), P D D$ at $5 \mathrm{~cm}, 10 \mathrm{~cm}, 15 \mathrm{~cm}$ and $20 \mathrm{~cm}$ beam were compared for selective field sizes $(5 \times 5,10 \times 10,15 \times 15$, $20 \times 20) \mathrm{cm}^{2}$. Although the dose per pulse is the same for varying dose rates, measurement is performed with a constant dose rate of $300 \mathrm{MU} / \mathrm{Min}$.

The least-square algorithm smoothened all percentage depth doses. After that PDDs were normalizing at a depth of maximum to $100 \%$. Point by point comparisons of the depth dose curve is performed up to a measurement scanning depth of $35 \mathrm{~cm}$. To analyze the beam dose profiles, they were normalized for all radiation field sizes and depths to $100 \%$ on the central beam axis. Figure 1 shows $6 \mathrm{MV}$ photon beam's dose profiles for a field size of $10 \times 10 \mathrm{~cm}^{2}$ at different depths on the central beam axis. It can be seen in Figure 1 that the dose profiles were depended on the depth on the central beam axis; the curves of the dose profiles were widened more to more as the depth increased due to an inverse square law (ISL) that it was the relationship between the depth and the beam divergence. Next, the dose profiles were graphed for the photon beam field sizes of $5 \times 5 \mathrm{~cm}^{2}, 10 \times 10 \mathrm{~cm}^{2}, 15 \times 15 \mathrm{~cm}^{2}, 20 \times 20 \mathrm{~cm}^{2}$ is shown in Figure 1.

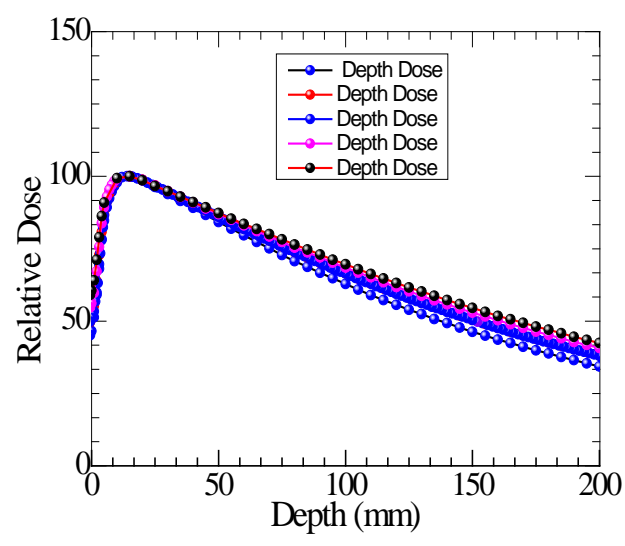

Figure 1. Graphical representation of PDD $(5 \times$ $5,10 \times 10,15 \times 15,20 \times 20)$ raw data. 
Table 1. Corresponding field sizes used for Output factors measurement in charge (nC).

\begin{tabular}{ccccccccccc}
\hline$\downarrow$ & $\rightarrow$ & $\mathrm{X}$ & & & & & & & & \\
\hline $\mathrm{Y}$ & 2 & 3 & 5 & 7 & 10 & 15 & 20 & 25 & 30 & 40 \\
\hline 2 & 9.45 & 9.77 & 9.97 & 10.1 & 10.2 & 10.28 & 10.33 & 10.36 & 10.37 & 10.39 \\
3 & 11.44 & 11.79 & 12.15 & 12.33 & 12.49 & 12.61 & 12.68 & 12.69 & 12.73 & 12.75 \\
5 & 11.9 & 12.39 & 12.9 & 13.2 & 13.45 & 13.64 & 13.73 & 13.79 & 13.84 & 13.85 \\
7 & 12.09 & 12.64 & 13.3 & 13.67 & 13.99 & 14.25 & 14.39 & 14.45 & 14.51 & 14.55 \\
10 & 12.26 & 12.87 & 13.62 & 14.1 & 14.50 & 14.85 & 15.02 & 15.13 & 15.20 & 15.26 \\
15 & 12.39 & 13.05 & 13.92 & 14.45 & 14.96 & 15.41 & 15.64 & 15.78 & 15.88 & 15.97 \\
20 & 12.48 & 13.18 & 14.07 & 14.67 & 15.22 & 15.73 & 16.01 & 16.19 & 16.32 & 16.42 \\
25 & 12.52 & 13.23 & 14.17 & 14.77 & 15.36 & 15.92 & 16.23 & 16.44 & 16.58 & 16.70 \\
30 & 12.57 & 13.29 & 14.22 & 14.86 & 15.48 & 16.05 & 16.39 & 16.64 & 16.79 & 16.91 \\
40 & 12.64 & 13.38 & 14.34 & 14.98 & 15.61 & 16.24 & 16.60 & 16.84 & 17.02 & 17.18 \\
\hline
\end{tabular}

The depth dose $\left(D_{\max }\right)$ at $10 \mathrm{~cm}$ depth $\left(D_{10}\right)$ for a $6 \mathrm{MV}$ photon beam for $5 \times 5$ $\mathrm{cm}^{2}$ FS was obtained from Figure 1 . The PDD at $10 \mathrm{~cm}$ depth $\left(D_{10}\right)$ is 62.8 , and $\mathrm{D}_{\max }$ is 100 . The maximum PDD (define the field size) $69.7 \%$, and the minimum $\mathrm{PDD}$ is $62.8 \%$. For a $6 \mathrm{MV}$ photon beam, the maximum value is $1.5 \mathrm{~cm}$.

The dose profile for $5 \times 5 \mathrm{~cm}^{2}$ FS and $10 \mathrm{~cm}$ depth SSD $=100 \mathrm{~cm}$ are shown in Figure 2. The lateral distance between $80 \%$ and $20 \%$ of the dose on the central beam axis, called semi-darkness, is for a $6 \mathrm{MV}$ photon beam. Table 2 shows Flatness and Symmetry for $5 \times 5 \mathrm{~cm}^{2} \mathrm{FS}$.

The field flatness changes with depth. This is attributed to an increase in scatter to primary dose ratio with increasing depth and decreasing incident photon energy off-axis, as shown in Figure 3. According to this the lateral distance between $80 \%$ and $20 \%$ of the dose on the beam central beam axis, called semi semi-darkness, is for a $6 \mathrm{MV}$ photon beam. The dose profile for $10 \times 10 \mathrm{~cm}^{2} \mathrm{FS}$ and $10 \mathrm{~cm}$ depth, SSD100 $\mathrm{cm}$. Table 3 shows Flatness and Symmetry for $10 \times 10$ $\mathrm{cm}^{2} \mathrm{FS}$.

It can be seen from Figure 4. that the normalized increase in relative dose curves presented the peaks at a depth of $10 \mathrm{~cm}$ for all field size over than field size of $15 \times 15 \mathrm{~cm}^{2}$. The peak values increased with field size, the increase in relative dose for the broad field size due to the scattered photon beam of $6 \mathrm{MV} \mathrm{Li}$ near accelerator. The flatness of photon beams is extremely sensitive to change in energy of the incident beam. A small change in the photon beam penetrative quality of a photon beam's penetrative quality results in a considerable change in the beam flatness. The field flatness changes with depth. This is attributed to an increase in scatter to the primary dose ratio with increasing depth and decreasing incident photon energy off-axis, as shown in Figure 4. According to this, the lateral distance between $80 \%$ and $20 \%$ of the dose on the central beam axes, called semi-darkness, is for a $6 \mathrm{MV}$ photon beam. The dose profile for $15 \times 15$ $\mathrm{cm}^{2} \mathrm{FS}$ and $10 \mathrm{~cm}$ depth SSD $=100 \mathrm{~cm}$. Table 4 shows Flatness and Symmetry for $15 \times 15 \mathrm{~cm}^{2} \mathrm{FS}$. 
The dose profile for $20 \times 20 \mathrm{~cm}^{2} \mathrm{FS}$ and $10 \mathrm{~cm}$ depth SSD100 $\mathrm{cm}$ are shown in Figure 5. Flatness and symmetry values for $6 \mathrm{MV}$ photon beam are listed in Table 5 .

The dose profile for diagonal Profile(C) $20 \times 20 \mathrm{~cm}^{2}$ FS and $10 \mathrm{~cm}$ depth SSD $=100 \mathrm{~cm}$ are shown in Figure 6. Flatness and symmetry values for $6 \mathrm{MV}$ photon beam are listed in Table 6.

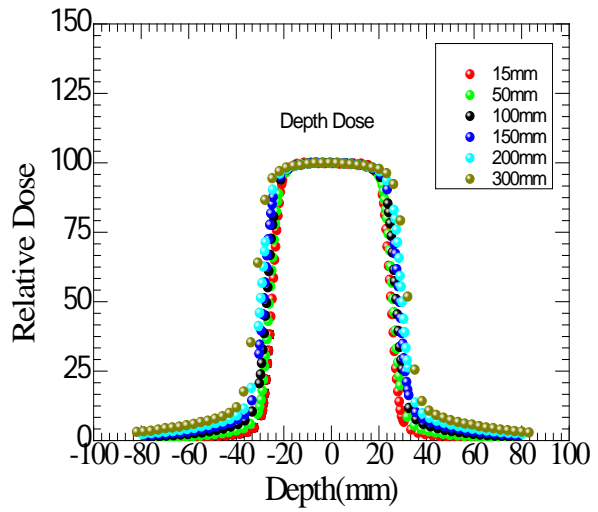

Figure 2. Graphical representation of 6 MVDose Profile $(C)$ for $5 \times 5$ field size raw data.

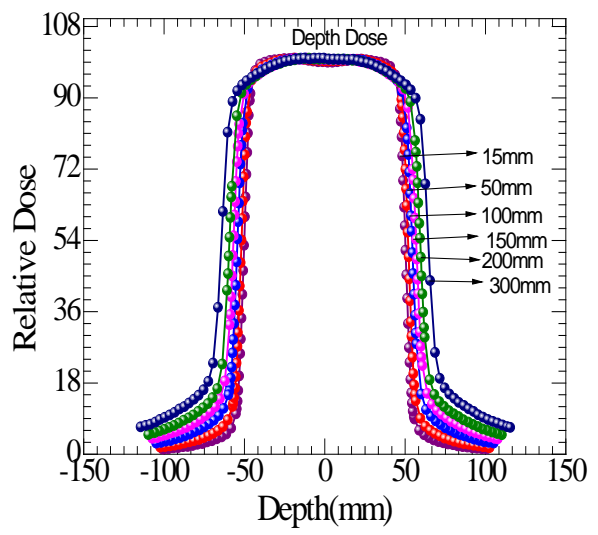

Figure 3. Graphical representation of 6 MVDose Profile(C) for $10 \times 10$ field size raw data.

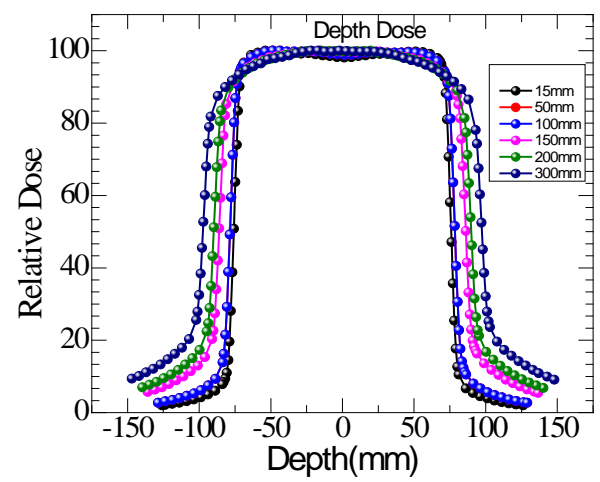

Figure 4. Graphical representation 6 MVDose Profile(C) for $15 \times 15$ field size raw data. 


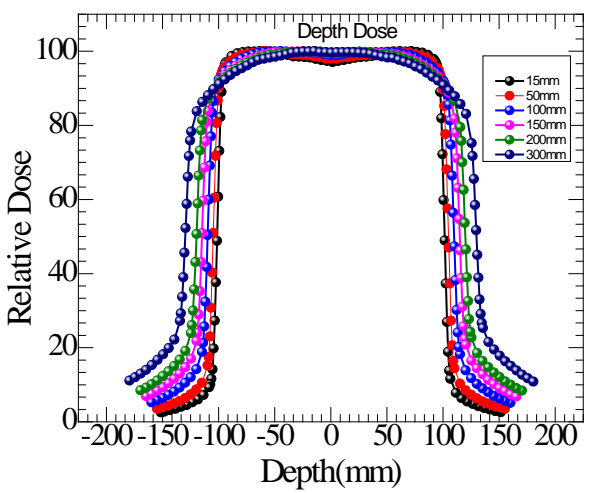

Figure 5. Graphical representation 6 MVDose Profile(C) for $20 \times 20$ field size raw data.

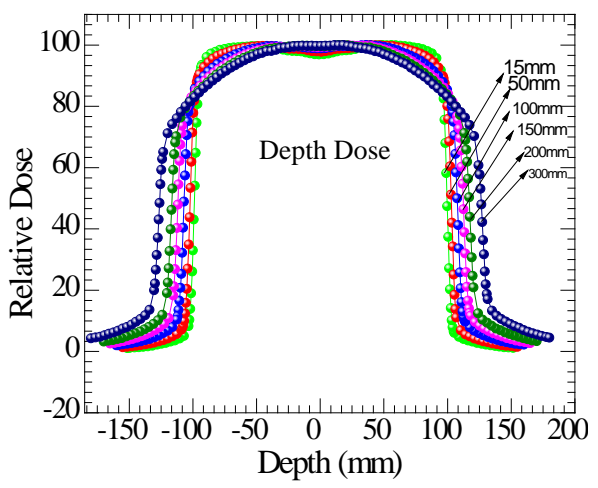

Figure 6. Graphical representation of diagonal Profile(C) for $20 \times 20$ raw data.

Table 2. Flatness and symmetry for $5 \times 5 \mathrm{~cm}^{2} \mathrm{FS}$.

\begin{tabular}{ccccc}
\hline Depth $(\mathrm{cm})$ & Max Value & Min Value & Flatness (\%) & Symmetry (\%) \\
\hline 1.5 & 99.1 & 85.3 & 7.4 & 2.64 \\
5 & 99.7 & 83.3 & 8.96 & 0.48 \\
10 & 99.6 & 82.2 & 9.71 & 0.98 \\
15 & 99.6 & 90.4 & 4.8 & 4.9 \\
20 & 99.7 & 93.6 & 3.1 & 1.79 \\
30 & 98.9 & 79 & 11.1 & 1.93 \\
\hline
\end{tabular}

Table 3. Flatness and symmetry for $10 \times 10 \mathrm{~cm}^{2} \mathrm{FS}$.

\begin{tabular}{ccccc}
\hline Depth $(\mathrm{cm})$ & Max Value & Min Value & Flatness (\%) & Symmetry (\%) \\
\hline 1.5 & 99 & 85.6 & 7.25 & 2.69 \\
5 & 99.7 & 83.7 & 8.72 & 2.19 \\
10 & 99.4 & 82.3 & 9.4 & 1.73 \\
15 & 99.7 & 87.6 & 6.46 & 3.91 \\
20 & 99.4 & 85.5 & 7.51 & 3.82 \\
30 & 99.7 & 84.6 & 8.19 & 1.98 \\
\hline
\end{tabular}


Table 4. Flatness and symmetry for $15 \times 15 \mathrm{~cm}^{2} \mathrm{FS}$.

\begin{tabular}{ccccc}
\hline Depth $(\mathrm{cm})$ & Max Value & Min Value & Flatness (\%) & Symmetry (\%) \\
\hline 1.5 & 99.1 & 83.4 & 8.6 & 1.45 \\
5 & 99.5 & 86.5 & 6.75 & 3.39 \\
10 & 97.7 & 84.2 & 7.42 & 1.44 \\
15 & 99.8 & 82.1 & 9.73 & 0.49 \\
20 & 99.7 & 83.5 & 8.84 & 1.27 \\
30 & 99.4 & 81.8 & 9.71 & 2.3 \\
\hline
\end{tabular}

Table 5. Flatness and symmetry for $20 \times 20 \mathrm{~cm}^{2} \mathrm{FS}$.

\begin{tabular}{ccccc}
\hline Depth $(\mathrm{cm})$ & Max Value & Min Value & Flatness (\%) & Symmetry (\%) \\
\hline 1.5 & 100 & 93.1 & 3.57 & 2.25 \\
5 & 99.8 & 86.2 & 7.31 & 5.25 \\
10 & 99.6 & 83.4 & 10 & 1.27 \\
15 & 99.6 & 80.9 & 10.36 & 1.82 \\
20 & 99 & 81.4 & 9.75 & 1.18 \\
30 & 99.6 & 83.8 & 8.61 & 0.42 \\
\hline
\end{tabular}

Table 6. Flatness and symmetry for $20 \times 20 \mathrm{~cm}^{2}$ FS diagonal Profile.

\begin{tabular}{ccccc}
\hline Depth $(\mathrm{cm})$ & Max Value & Min Value & Flatness (\%) & Symmetry (\%) \\
\hline 1.5 & 99.6 & 82.5 & 9.39 & 1.10 \\
5 & 98.5 & 83.6 & 8.18 & 3.56 \\
10 & 99.8 & 81.4 & 10.15 & 2.13 \\
15 & 99.7 & 82.9 & 9.2 & 0.85 \\
20 & 99.5 & 80 & 10.86 & 0.43 \\
30 & 99 & 81.6 & 9.63 & 2.12 \\
\hline
\end{tabular}

\section{Conclusions}

The process of commissioning an LINAC for clinical use includes comprehensive measurements of dosimetric parameters where a full set of data is acquired that will be used for patient treatment planning. The essential parameters for single energy that include percentage depth dose (PDD), Open Beam Profiles (Inline, Crossline \& Diagonal), and Beam Output factors for a series of Field Sizes (FSs) are the essential dosimetric data that require during the commissioning period of a medical linear accelerator (LINAC). Since the outcome of radiation treatment is directly related to the precession in the delivered dose to the patient, it depends on the accuracy of beam data used in the treatment planning process. Therefore, to operate an LINAC for treatment purposes, the measurement of percentage depth (PDD) is very crucial. The differences of flatness and symmetry contamination at $6 \mathrm{MV}$ beams were less than $\pm 2 \%$ to $\pm 3 \%$, re- 
spectively.

We can also analyze the golden beam data (machine first beam data which is measured by the manufacture company at the time of machine assemble) only if we had it. Then we can compare it with the measured beam data and can investigate any disagreement among them. The photon beam dosimetry data is found in current study among the linear accelerators with the same model and nominal energy. All tolerances were within the clinically acceptable tolerance limit. From clinical point of view, this study will support the radiation oncology department to avoid any delay or interruption in the treatment of patients. The PDD, Dose profile, Output Factors, beam flatness and symmetry data obtained for $6 \mathrm{MV}$ photon energies in this work will be useful for initial commissioning of LINAC system. Its use for minimum error calculation and greater accuracy will be investigated in future works.

\section{Acknowledgements}

The authors would like to thank all the personnel of the Institute of Nuclear Medical Physics (INMP), Bangladesh Atomic Energy Commission for allowing us to use the VARIAN Clinac iX and give necessary support for this work.

\section{Conflicts of Interest}

The authors declare no conflicts of interest regarding the publication of this paper.

\section{References}

[1] Linear Accelerator. http://www.radiologyinfo.org/en/info.cfm?pg=linac

[2] Mayles, P., Nahum, A. and Rosenwald, J.C. (2007) Handbook of Radiotherapy Physics Theory and Practice. CRC Press, Boca Raton, 452. https://doi.org/10.1201/9781420012026

[3] Chang, D.S., lasley, F.D., DAS, I.J., Mendonca, M.S. and Dynlacht, J.R. (2014) Dosimetry of Photon Beams in Water. In: Basic Radiotherapy Physics and Biology, Springer, New York, 77. https://doi.org/10.1007/978-3-319-06841-1_8

[4] International Atomic Energy Agency (2000) Absorbed Dose Determination in External Beam Radiotherapy. Technical Reports Series No. 398, Vienna, 110.

[5] Das, I.J., et al. (2008) Accelerator Beam Data Commissioning Equipment and Procedures. Medical Physics, 35, 4186-4215. https://doi.org/10.1118/1.2969070

[6] Tuathan, O. (2011) Monte Carlo Simulation of Medical Accelerator Electron Treatment Heads. Thesis, School of Physics, National University of Ireland Galway, Ireland.

[7] Podgorsak, E.B. (2010) Radiation Physics for Medical Physicists. Springer, Heidel berg, 277. https://doi.org/10.1007/978-3-642-00875-7

[8] Kim, S., Liu, C.R., Zhu, T.C. and Palta, J.R. (1998) Photon Beam Skin Dose Analyses for Different Clinical Setups. Medical Physics, 25, 860-866.

https://doi.org/10.1118/1.598261 